\title{
Mechanical response of active gels
}

\author{
T. B. Liverpool ${ }^{1}$, M. C. Marchetti ${ }^{2}$, J-F. Johnny ${ }^{3}$ and J. Prost ${ }^{4}$ \\ 1 Department of Mathematics, University of Bristol, University Walk, Clifton, Bristol BS8 1TW, U.K. \\ 2 Physics Department and Syracuse Biomaterials Institute, Syracuse University, Syracuse, NY 13244, USA. \\ ' 3 Physicochimie Curie (CNRS-UMR168), Institut Curie, Section de Recherche, 26 rue d'Ulm, 75248 Paris Cedex 05, \\ France. \\ 4 E.S.P.C.I., 10 rue Vauquelin, 75231 Paris Cedex 05, France.
}

\begin{abstract}
PACS nn.mm.xx - First pacs description
PACS $\mathrm{nn} \cdot \mathrm{mm} . \mathrm{xx}$ - Second pacs description

PACS $\mathrm{nn} . \mathrm{mm} . \mathrm{xx}-$ Third pacs description
\end{abstract}

\begin{abstract}
We study a model of an active gel of cross-linked semiflexible filaments with additional active linkers such as myosin II clusters. We show that the coupling of the elasticity of the semiflexible filaments to the mechanical properties of the motors leads to contractile behavior of the gel, in qualitative agreement with experimental observations. The motors, however, soften the zero frequency elastic constant of the gel. When the collective motor dynamics is incorporated in the model, a stiffening of the network at high frequencies is obtained. The frequency controlling the crossover between low and high frequency network elasticity is estimated in terms of microscopic properties of motors and filaments, and can be as low as $10^{-3} \mathrm{~Hz}$.
\end{abstract}

Introduction. - The mechanical properties of cells control many biological functions, including the sensing and generation of forces, cell motility and cell division. The response of the cell to mechanical stimuli is mediated by the cytoskeleton, a network of semiflexible filaments (F-actin, microtubules and intermediate filaments) linked ' by a variety of passive and active proteins. [1,2] The cytoskeleton is maintained out of equilibrium by chemical reactions that drive force generation by motor proteins, as , well as by filament treadmilling. A variety of recent experiments have measured the remarkable rheological properties of this intrinsically nonequilibrium polymer network. These include bulk and microrheology of in vitro stabilized networks of cytoskeletal filaments with a controlled concentration of various crosslinkers, as well as in vivo whole cell rheology.

Cross-linked entangled actin networks are viscoelastic solids, with a time-dependent mechanical response (stress $\sigma$ ) to deformation (strain $\gamma$ ). These networks have both viscous and elastic responses characterized by loss $G^{\prime \prime}(\omega) \sim \sigma / \dot{\gamma}$ and storage moduli $G^{\prime}(\omega) \sim \sigma / \gamma$, respectively. For cross-linked gels, the elastic (storage) modulus dominates the mechanical response and reaches a frequency independent plateau $G_{0}$ at low frequencies (less than $1 \mathrm{~Hz}$ ). Experimentally $G_{0}$ is found to depend strongly on cross-link density and can vary from 0.1 - $100 \mathrm{~Pa}$ [3]. For frequencies above $1 \mathrm{~Hz}$, both the storage and loss moduli show a high frequency behavior $G^{\prime}, G^{\prime \prime} \sim \omega^{3 / 4}$ characteristic of semiflexible polymer dynamics [4].

Measurements of the mechanical properties of cells yield, however, quite different behaviour [7]. The low frequency $(<10 \mathrm{~Hz})$ shear moduli are observed to behave as, $G^{\prime}, G^{\prime \prime} \sim G_{*}\left(\omega / \omega^{*}\right)^{\alpha}$, with a small exponent $\alpha \sim 0.15-0.2, G_{*} \sim 10^{2}-10^{3} \mathrm{~Pa}$ and $\omega^{*} \sim 1 \mathrm{~Hz}[8-13]$. Significantly, the magnitude of $G_{*}$ is much higher than the typical plateau moduli of purified in-vitro actin gels. While increasing cross-linker density can significantly enhance the elastic modulus [3], it is surprising that it would have such a dramatic effect on the loss modulus. It was recently suggested that the remarkable stiffening of the low frequency linear response of active gels may be due to the internal stresses generated by the presence of active crosslinkers, such as myosin II minifilaments [14,15]. Recent quantitative experiments studying the mechanics of in-vitro networks of F-actin, with passive ( $\alpha$-actinin) and active (muscle myosin II) cross-linkers, have shown both stiffening [14] and contractile behaviour [17] of these reconstituted networks. Interestingly the contractile behaviour has been shown to appear only in a narrow concentration range of passive cross-linkers. 
In this letter we present a theoretical description of active gels which can explain both the contractile behaviour and the intermediate-frequency stiffening of these systems. The minimal element from which the active gel is constructed is a pair of filaments cross-linked by an active cluster of molecular motors. This has been a useful starting point for explaining the properties of soft active materials in both the fluid [18] and the gel phase [15]. Our work shows that the coupling of the elasticity of the semiflexible filaments to the motor dynamics plays a crucial role in controlling the rheology of the network. We find that active clusters lead to contraction of the gel which has a more dense ground state than a gel with the active crosslinks replaced by passive ones. The zero frequency stiffness of our model active gel, when perturbed from this ground state, is lower than that of the corresponding passive gel, in apparent contradiction with experiments. However, at higher frequencies the collective dynamics of the motors stiffens the gel as compared to the passive case. This qualititive behaviour is obtained both in the regime of linear chain elasticity and when taking account of nonlinearities.

Model. - We consider an ideal semiflexible polymer network with both permanent and active crosslinkers. The network consists of isotropically oriented stiff polymer segments of length shorter than their persistence length subjects to rigid constraints due to the permanent crosslinks. The motor clusters act as dynamic cross-linkers which apply equal and opposite forces to pairs of filaments. We do not consider the effect of entanglements.

We parametrize each filament by a curve $\mathbf{R}(s)$, with $0 \leq s \leq L$ and $L$ the contour length. We consider small deviations from a straight configuration of the polymer segment and decompose deformations of length scales smaller than the persistence length $L_{p}=\kappa / k_{B} T$, with $\kappa$ the bending rigidity, in transverse and longitudinal components by writing $\mathbf{R}(s)=R_{\|}(s) \hat{\mathbf{u}}+\mathbf{r}_{\perp}(s) \equiv\left[s-r_{\|}(s)\right] \hat{\mathbf{u}}+\mathbf{r}_{\perp}(s)$, where $\hat{\mathbf{u}}$ is a unit vector giving the orientation of the segment and $\mathbf{r} \cdot \hat{\mathbf{u}}=0[16]$. In a cross-linked gel, the free energy of each filament is given by

$$
F=\int_{0}^{L} d s\left\{\frac{\kappa}{2}\left|\partial_{s}^{2} \mathbf{R}\right|^{2}-\sigma_{0} \hat{\mathbf{u}} \cdot \partial_{s} \mathbf{R}\right\} .
$$

The first term is the usual bending energy of a worm-like chain and the second one describes the tension $\sigma_{0}$ of the filaments due the permanent crosslinkers. The filament satisfies the boundary conditions $\mathbf{R}(0)=0, \mathbf{R}(L)=L_{0} \hat{\mathbf{u}}$ and $\left[\partial_{s}^{2} \mathbf{R}(s)\right]_{s=0}=\left[\partial_{s}^{2} \mathbf{R}(s)\right]_{s=L}=0$, where $L_{0}$ is the distance between fixed (passive) crosslinks [19]. Transverse and longitudinal deviations are coupled by the constraint of inextensibility, $\partial_{s} r_{\|}=\frac{1}{2}\left|\partial_{s} \mathbf{r}_{\perp}\right|^{2}+\mathcal{O}\left(\left|\partial_{s} \mathbf{r}_{\perp}\right|^{4}\right)[16]$.

The effective longitudinal response function of a filament is evaluated by averaging over the transverse fluctuations, with the result

$$
\left\langle\partial_{s} r_{\|}\right\rangle_{0}=\frac{L}{L_{p}}\left\{\frac{x \operatorname{coth} x-1}{x^{2}}\right\} \equiv \mathcal{F}\left(L, \sigma_{0}, \kappa\right),
$$
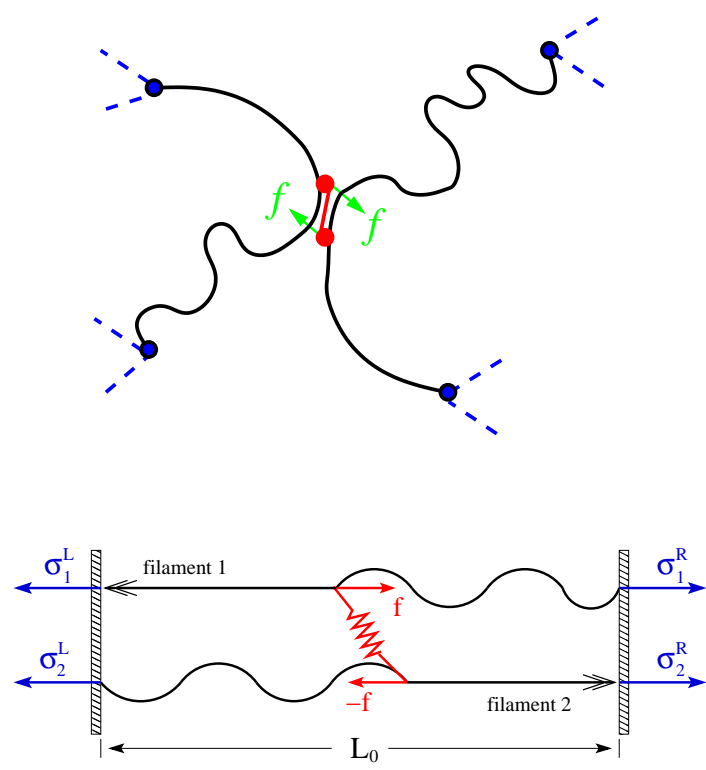

Fig. 1: Top: A schematic representation of two semiflexible filaments crosslinked by an active myosin cluster (shown in red) and linked to neighboring filaments (blue dashed lines) by passive linkers (blue dots). Bottom: The minimal elastic element of our active gel, consisting of two antiparallel filaments crosslinked by a motor cluster. The motor cluster is modeled as a spring of stiffness $k_{m}$ that exerts equal and opposite forces of magnitude $f$ on the two filaments. The pair of filaments is maintained at a fixed distance $L_{0}$ by passive crosslinkers. The asymmetric shape of each filament indicates that due to their nonlinear elastic properties the filaments are easier to compress than to stretch.

and $x=L \sqrt{\frac{\sigma_{0}}{\kappa}}$. The end-to-end length of the filament is

$$
L_{0}=L-\int_{0}^{L} d s\left\langle\partial_{s} r_{\|}\right\rangle_{0}=L-\left[\left\langle r_{\|}(L)\right\rangle_{0}-\left\langle r_{\|}(0)\right\rangle_{0}\right]
$$

In the limit $\sigma_{0} \rightarrow 0$ the filament is roughened by thermal fluctuations and $\mathcal{F} \sim \frac{k_{B} T L}{6 \kappa}\left[1-x^{2} / 15\right]$ for $x \ll 1$. Conversely, for $x \gg 1 \mathcal{F} \sim k_{B} T / 2 \sqrt{\kappa \sigma_{0}}$ and all wrinkles are pulled out by the applied tension so that $L=L_{0}$.

Now we consider the effect of additional active crosslinks on the mechanical properties of the gel. If the gel is kept under constant external tension, the activity of the motors changes the end-to-end distance $L_{0}$. Conversely, if $L_{0}$ is changed by applying an external deformation, the active cross-links induce an additional tension on the filaments. Both types of response may be studied by evaluating the change in extension of the filaments upon increasing of the local tension from $\sigma_{0}$ to $\sigma_{0}+\sigma(s)$. The additional tension may be thought of as arising either from motor activity or from externally applied forces. To describe this response we define an 'elastic' deformation field $u(s)=\left[\left\langle R_{\|}(s)\right\rangle-\left\langle R_{\|}(s)\right\rangle_{0}\right]$, where $\left\langle R_{\|}(s)\right\rangle$ denotes the effective longitudinal response to the total tension $\sigma_{0}+\sigma$. In the limit of large $\sigma_{0}$, this yields a general relationship 
between the deformation and the tension,

$$
\partial_{s} u(s)=G\left[\sigma_{0}, \sigma(s)\right],
$$

with $G=\mathcal{F}\left(L, \sigma_{0}, \kappa\right)-\mathcal{F}\left(L, \sigma_{0}+\sigma(s), \kappa\right)$. Eq. (4) describes the nonlinear elasticity of a semiflexible filament under tension and is the starting point of our analysis.

The top image of Fig. 1 shows a schematic of a motor cluster crosslinking two semiflexible filaments, which are in turn bound at their ends by permanent crosslinks. The motors in the cluster walk towards the plus end of each filament indicated by the double arrows, exerting equal and opposite forces $\pm f$ on the two filaments $[14,15,18]$, resulting in additional tension. Such a crosslinked filament pair is the fundamental elastic unit in our model of an active gel. For an isotropic gel, with uncorrelated orientations of filament pairs and motor clusters, the elastic properties of the gel may be obtained by suitable angular averages. All the essential physics can, however, be obtained from the simplified one-dimensional model depicted in the bottom part of Fig. 1. Taking account of the orientation of the filaments only changes the numerical prefactors. Also we consider only anti-parallel filaments as motors do not generate significant tensions on pairs of parallel filaments. Our goal is to calculate how the end-to-end distance and the mechanical response of the filament pair are changed by the presence of the active crosslink. The force balance equations for the two filaments are

$$
\sigma_{1}^{R}-\sigma_{1}^{L}+f=0, \quad \sigma_{2}^{R}-\sigma_{2}^{L}-f=0,
$$

where $\sigma_{i}^{R, L}$, for $i=1,2$ are the additional tensions (on top of the "bare" tension $\sigma_{0}$ ) at the ends of each filament due to active cross-links and/or external forces (Fig. 1).

Motor clusters. - We consider the response of the system on time scales long compared to the relaxation time of the longitudinal modes of the filaments so that we can ignore the dynamics of the deformation, $u(s)$. We include, however, the finite-frequency response of the motors, which is important when considering the response of the gel to frequency-dependent deformations. To do this we use the model introduced by Jülicher and Prost [20], where a motor cluster actively sliding along a polar filament is described as a collection of $N$ motors rigidly attached to a backbone and moving along a polar periodic track. Under the action of both thermal and ATP-driven excitations, each motor in the cluster undergoes transitions between a strongly bound and a weakly bound state. We model our active cross-link as two such motor clusters linked by a spring of stiffness $k_{m}$ that couples the motion along the top and bottom filaments. We denote by $s_{i}$ the position of the cluster along the $i$-th filament and by $u_{i}=u\left(s_{i}\right)$ the elastic deformation of the $i$-th filament at that point and assume that the center of mass position $s_{0}=\left(s_{1}+s_{2}\right) / 2$ of the motor cluster does not move.

The dynamics of the points of attachment of the motor cluster interacting with the filaments is described by the force balance equations

$$
\zeta_{0} \partial_{t} s_{1}=-\zeta_{0} \partial_{t} s_{2}=-k_{m}\left(s_{1}-s_{2}+u_{1}-u_{2}\right)-f,
$$

where $\zeta_{0}$ is a friction. The first term on the right hand side of Eq. (6) represents elastic forces within the motor cluster. In the last term, $-f$ is the force exerted by the motors on filament 1 . In a steady state, where the velocity of the motor cluster vanishes, $f$ equals the stall force $f_{s}$. As in Ref. [20], if the motor clusters are not exactly at stall force we expand the force up to first order in the motors velocity and introduce an active frequency-dependent friction $\zeta_{a c t}(\omega)$ on the motors, which can be negative. For a sake of simplicity, we will assume here that the motor clusters have no spontaneous oscillations, which is the case if the ATP concentration is low enough.

Static response. - We first examine the static response of a cross-linked filament pair to a change in the end-to-end distance $L_{0}$. We apply a force $2 F$ to the filament pair and calculate the resultant extension of the right-hand side of both filaments $\Delta(F)$. A value $\Delta(0)<0$ at zero applied force corresponds to contractile behaviour.

The conformation of each filament is described by a displacement $u_{i}(s)$, with $\partial_{s} u_{i}=G\left[\sigma_{0}, \sigma_{i}(s)\right]$. These equations must be solved with with boundary conditions $u_{i}(0)=0$ and $u_{i}(L)=\Delta$, for $i=1,2$. Force balance on the filaments indicate that the tension has a jump discontinuity at the point of motor attachment. This implies that the derivative $\partial_{s} u_{i}(s)$ is also piecewise constant, with a jump discontinuity of magnitude $f$ at $s_{i}$. Requiring the displacement of each filament $u_{i} \equiv u_{i}\left(s_{i}\right)$ at the point of attachment to be continuous, we obtain

$$
\begin{aligned}
\frac{u_{i}}{s_{i}} & =G\left[\sigma_{0}, \sigma_{0}+\sigma_{i}^{L}\right], \\
\frac{\Delta-u_{i}}{L-s_{i}} & =G\left[\sigma_{0}, \sigma_{0}+\sigma_{i}^{R}\right],
\end{aligned}
$$

for $i=1,2$. Eliminating $u_{1}$ and $u_{2}$ from Eqs. (7) and (8), and from the stall condition, $f_{s}=f=-k_{m}\left(\Delta s+u_{1}-u_{2}\right)$, we obtain

$$
\begin{aligned}
& s_{1} G\left(\sigma_{1}^{L}\right)+\left(L-s_{1}\right) G\left(\sigma_{1}^{R}\right)=\Delta, \\
& s_{2} G\left(\sigma_{2}^{L}\right)+\left(L-s_{2}\right) G\left(\sigma_{2}^{R}\right)=\Delta, \\
& \left(s_{1}-s_{2}\right)+s_{1} G\left(\sigma_{1}^{L}\right)-s_{2} G\left(\sigma_{2}^{L}\right)=-\frac{f}{k_{m}},
\end{aligned}
$$

where $G(\sigma) \equiv G\left[\sigma_{0}, \sigma_{0}+\sigma\right]$. From the two force balance equations, Eqs. (5), it is evident that only two of the four tensions $\sigma_{i}^{R, L}$ are independent. It is convenient to eliminate two of the unknowns by introducing new forces $F_{i}=\frac{1}{2}\left(\sigma_{i}^{R}+\sigma_{i}^{L}\right)$, so that the force balance equations are automatically satisfied. Letting $s_{1,2}=s_{0} \pm \Delta s / 2$, $F=\left(F_{1}+F_{2}\right) / 2$ and $\delta F=F_{1}-F_{2}$, Eqs. (9)[11) yield a set of three coupled equations in three unknown $\Delta s, \delta F$ and either $\Delta$ of $F$. The equations can be solved to obtain either the displacement $\Delta$ as a function of the total force, 
$\Delta(F)$, or $F(\Delta)$. The solution will depend parametrically on the center of mass position of the motor cluster, $s_{0}$.

We solve Eqs. (91), (10) and (11) taking into account the nonlinear elasticity of the filaments, in the perturbative limit where all the motor induced forces are small compared to the bare tension $\sigma_{0}$ and solve the equations perturbatively in $f$. If all tensions $\sigma_{i}^{R, L}$ are small compared to $\sigma_{0}$ one can approximate $G\left[\sigma_{0}, \sigma\right]=\sum_{n} G^{n} \sigma^{n} / n ! \approx$ $G^{\prime} \sigma+G^{\prime \prime} \sigma^{2} / 2+G^{\prime \prime \prime} \sigma^{3} / 6+\ldots$, where $G^{n} \equiv G^{n}\left(\sigma_{0}\right)=$ $\left(\frac{\partial^{n} G\left[\sigma_{0}, \sigma_{0}+\sigma\right]}{\partial \sigma^{n}}\right)_{\sigma=0}$, with $G^{\prime}=\left(\partial G\left[\sigma_{0}, \sigma\right] / \partial \sigma\right)_{\sigma=0}$, etc .

To linear order in the total force $F$, we write

$$
\Delta(F)=\Delta(0)+\frac{F}{k_{\mathrm{eff}}}+\mathcal{O}\left(F^{2}\right) .
$$

The ground state deformation is given by

$$
\frac{\Delta(0)}{L}=-\frac{f^{2}}{2}\left[\frac{G^{\prime}}{k_{m} L}-\phi(1-\phi)\left(G^{\prime \prime}-2\left(G^{\prime}\right)^{2}\right)\right],
$$

where $\phi=s_{0} / L$. Since $G^{\prime}\left(\sigma_{0}\right)>0$ and $G^{\prime \prime}\left(\sigma_{0}\right)<0$ the ground state deformation is always negative, corresponding to a contractile system. This result is easily understood if we consider the limit where $G^{n}=0$ for $n>1$ and the filaments behave as a linear springs of elastic constant $k_{0}=\left(L G^{\prime}\right)^{-1}$. In this case the ground state deformation can be written as $\Delta=f G^{\prime} \Delta s_{0} / 2$, where $\Delta s_{0}=-\frac{f}{k_{m}}-2 \phi(1-\phi) \frac{f}{k_{0}}$ is the ground state value of separation of the motor clusters between the two filaments. Then $\Delta$ is easily obtained by equating the change in elastic energy when the filaments are stretched from $L$ to $L+\Delta$, given by $2\left[\frac{1}{2} k_{0}(L+\Delta)^{2}-\frac{1}{2} k_{0} L^{2}\right] \simeq 2 k_{0} L \Delta$ to the work $f \Delta s_{0}$ done by the motor clusters on the filaments. The effective stiffness of the network is given by

$$
\begin{aligned}
\frac{1}{k_{\mathrm{eff}}}= & \frac{1}{k_{0}}+L f^{2} \phi(1-\phi)\left[G^{\prime \prime \prime} / 2-2 G^{\prime} G^{\prime \prime}+\left(G^{\prime}\right)^{3}\right] \\
& -\frac{f^{2}}{2 k_{m}}\left[G^{\prime \prime}+\left(G^{\prime}\right)^{2}\right] .
\end{aligned}
$$

The average ground state deformation and the effective stiffness of the element are shown in Fig. 2 as functions of $L$, with $\overline{\Delta(F)}=\int_{0}^{1} d \phi \Delta(F)$. Contractile behaviour is observed for all $L$ and vanishes as $L \rightarrow 0$, reflecting the higher resistance of short filaments to compression. On the other hand, the active crosslinks always decrease the zero frequency stiffness of the gel which vanishes as $L \rightarrow \infty$.

Our perturbative analysis captures the qualitative experimental observation of contractile behaviour, but yields softening of the gel in contradiction with experiments. To address this we incorporate in the next section the finite frequency response of the motor clusters.

Finite frequency behaviour. - We now consider the finite frequency behaviour near stall and take into account the collective dynamics of the motors within an active crosslink cluster. We consider time-scales long compared to the relaxation time of the filaments, but include the finite-frequency response of the motors.
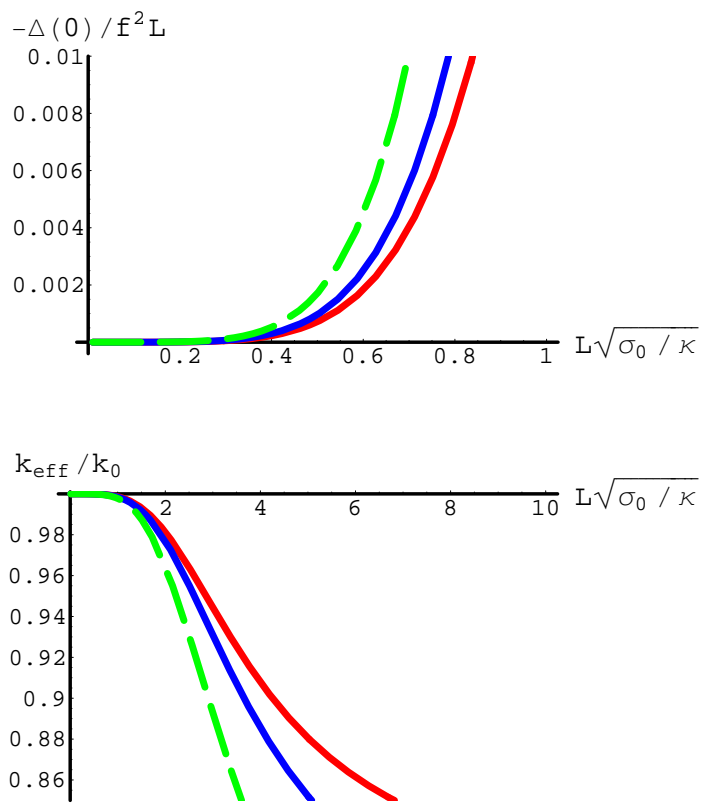

Fig. 2: Plot of $-\overline{\Delta(0)} / f^{2} L$ (top) and zero frequency stiffness $\overline{k_{\text {eff }}}$ (bottom) for $1 / k_{m}=0, \frac{f}{\sigma_{0}}=0.4$ as a function of $L \sqrt{\sigma_{0} / \kappa}$ for 3 values of $L_{p} \sqrt{\sigma_{0} / \kappa}=0.2$ (red), 0.17 (blue), 0.13 (green).

We apply an oscillatory deformation to the end-points of the right side of the filaments, $\Delta=\Delta_{0}+\epsilon \Delta_{1}(t)$, while keeping the left side fixed. Here $\Delta_{0} \equiv \Delta(0)$ is the static displacement at zero external force, corresponding to the motors' stall force. This perturbation will result in motors sliding along the filaments. To discuss the response of the system we work in Fourier space and linearize in all the deviations $f \rightarrow f+\delta f_{\omega}, \Delta s \rightarrow \Delta s+\delta s_{\omega}, F \rightarrow$ $F_{\omega}, \delta F \rightarrow \delta F+\delta F_{\omega}$, where $f, \Delta s, F$ and $\delta F$ denote static quantities at stall condition as defined earlier. From equation (6) the dynamics of the relative displacements of the two motor cluster's heads is given by

$$
\begin{aligned}
i \omega \zeta_{0} \delta s_{\omega}= & -2 k_{m}\left\{1+\frac{1}{2}\left[G\left(\sigma_{1}^{L}\right)+G\left(\sigma_{2}^{L}\right)\right]\right\} \delta s_{\omega}-2 \delta f_{\omega} \\
& -2 k_{m}\left[s_{1} \delta \sigma_{1 \omega}^{L} G^{\prime}\left(\sigma_{1}^{L}\right)-s_{2} \delta \sigma_{2 \omega}^{L} G^{\prime}\left(\sigma_{2}^{L}\right)\right]
\end{aligned}
$$

where $\delta \sigma_{1 \omega}^{L}=F_{\omega}+\frac{1}{2}\left(\delta F_{\omega}+\delta f_{\omega}\right)$ and $\delta \sigma_{2 \omega}^{L}=F_{\omega}-\frac{1}{2}\left(\delta F_{\omega}+\right.$ $\delta f_{\omega}$ ). Solving equations (78) and using the fact that the collective dynamics of the motors induces an active friction [20] so that $i \omega \zeta_{a c t}(\omega) \delta s_{\omega} / 2=\delta f_{\omega}$, we can obtain a linear relationship between $\delta s_{\omega}$ and $F_{\omega}$ and hence a frequency dependent correction to $k_{\text {eff. }}$. The expression is complicated but simplifies in the experimentally relevant regime of stiff motors $k_{m} \rightarrow \infty$ with the result

$$
\begin{aligned}
\frac{1}{k_{\mathrm{eff}}(\omega)}=\frac{1}{k_{\mathrm{eff}}(0)}+ & \frac{i \omega L f^{2} \phi(1-\phi)}{\omega_{c}-i \omega}\left[\frac{\left(G^{\prime \prime}\right)^{2}}{G^{\prime}}\right. \\
& \left.-3 G^{\prime} G^{\prime \prime}+2\left(G^{\prime}\right)^{3}\right],
\end{aligned}
$$


where $\omega_{c} \simeq\left(\zeta_{a c t} G^{\prime} L\right)^{-1}$. For $\omega \gg \omega_{c}$ we obtain an enhancement of the effective stiffness of the elastic element:

$$
\begin{aligned}
\frac{1}{k_{\mathrm{eff}}(\infty)} \simeq \frac{1}{k_{\mathrm{eff}}(0)}- & L f^{2} \phi(1-\phi)\left[\frac{\left(G^{\prime \prime}\right)^{2}}{G^{\prime}}\right. \\
& \left.-3 G^{\prime} G^{\prime \prime}+2\left(G^{\prime}\right)^{3}\right] .
\end{aligned}
$$

The effective low and high frequency elastic constants are shown in Fig. 3, Active cross-links always soften the zero frequency stiffness $k_{e f f}(0)$ of the elastic element, but at the same time always increase $k_{e f f}(\infty)$ relative to the stiffness $k_{0}$ of a single filament. An estimate of the crossover frequency $\omega_{c}$ suggests that the stiffening may be relevant at the intermediate frequencies probed in experiments. From [20] we estimate $\zeta_{\text {act }} \sim N \frac{1}{l^{2}} \frac{\omega_{2}\left(W_{2}-W_{1}\right)}{\left(\omega_{1}+\omega_{2}\right)^{2}}$, where $N$

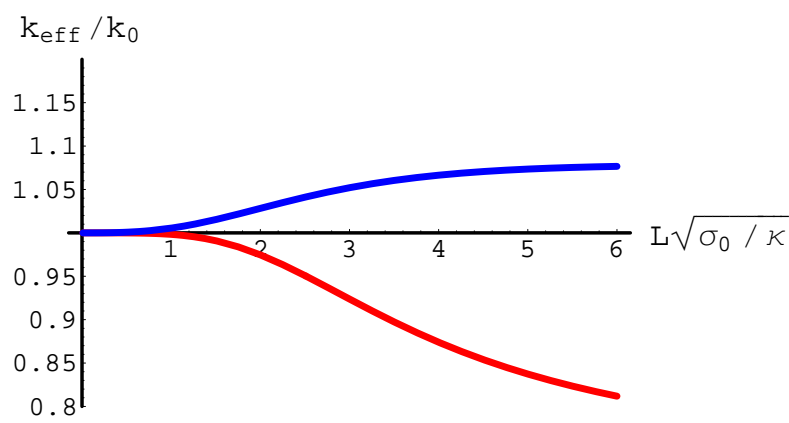

Fig. 3: Plot of $k_{\text {eff }}(0)$ (red online) and $k_{\text {eff }}(\infty)$ (blue online) for $1 / k_{m}=0, \frac{f}{\sigma_{0}}=0.4$ as a function of $L \sqrt{\sigma_{0} / \kappa}$ for $L_{p} \sqrt{\sigma_{0} / \kappa}=0.2$.

is the cluster size, $W_{1,2}$ are the typical potentials, $\omega_{1,2}$ typical motor conformation transition frequencies and $l$ the motor's step length. From the expression for $G$ above we estimate (at $\left.\sigma_{0} \simeq 0\right), G^{\prime}=\frac{L^{3}}{90 k_{B} T L_{p}^{2}}$. Finally we obtain $\omega_{c} \sim \frac{90}{N} \frac{\left(\omega_{1}+\omega_{2}\right)^{2}}{\omega_{2}}\left(\frac{k_{B} T}{W_{2}-W_{1}}\right)\left(\frac{L_{p}}{L}\right)^{2}\left(\frac{l}{L}\right)^{2}$. Using $l \simeq 4 \mathrm{~nm}$, $W_{2}-W_{1} \simeq 10 k_{B} T, L_{p} \simeq 20 \times 10^{3} \mathrm{~nm}, L \simeq 4 \mu \mathrm{m}, N \simeq 100$, and $\omega_{1,2} \simeq 100 \mathrm{~Hz}$, we obtain $\omega_{c} \sim 10^{-3} \mathrm{~Hz}$.

We found that both the contractility and the stiffening of the active element are proportional to the square of the stall force of the motor cluster. This point and its implication for the macroscopic shear modulus of a crosslinked network merit some discussion.

Shear modulus of an active gel. - First we use standard methods to relate the shear modulus of a crosslinked network of noninteracting elastic elements to the stiffness $k_{\text {eff }}$ of each elastic element $[5,6]$. We describe the active gel as a cross-linked semiflexible polymer gel of monomer density $\rho$, where each monomer is a sphere of diameter $a \ll L_{p}$. The mesh-size is then $\xi^{2}=1 /(\rho a)[4-6]$. Under a uniform shear, a point $\mathbf{x}$ of the network is deformed according to $\mathbf{x} \rightarrow \mathbf{x}+\mathbf{a}(\mathbf{x})$, with $\gamma_{i j}=\frac{1}{2}\left(\partial_{i} a_{j}+\partial_{j} a_{i}\right)$ the applied strain. An elastic segment of orientation $\hat{\mathbf{u}}$ and end-to-end distance $L_{0}$ undergoes a relative change $\delta L_{0} / L_{0}=\gamma_{i j} \hat{u}_{i} \hat{u}_{j}$. This deformation will in turn induce a tension $\sigma_{s}=k_{\text {eff }} \delta L_{0}$ in the elastic unit, where $1 / k_{\text {eff }}$ is the longitudinal response function of the unit. The corresponding contribution to the stress tensor of the gel is $\sigma_{i j}=\xi^{-2}\left\langle\sigma_{s} \hat{u}_{i} \hat{u}_{j}\right\rangle$, where $\langle\ldots\rangle$ denotes an average over the filaments' orientation. For an isotropic filament distribution $\left\langle\hat{u}_{i} \hat{u}_{j} \hat{u}_{k} \hat{u}_{l}\right\rangle=$ $(1 / 15)\left[\delta_{i j} \delta_{k l}+\delta_{i k} \delta_{j l}+\delta_{i l} \delta_{j k}\right]$. Defining the shear modulus $E$ of the gel via $\sigma_{i j}=2 E \gamma_{i j}[5,6]$, for an incompressible gel we obtain $E=\frac{1}{15} \xi^{-2} L_{0} k_{\text {eff }}$.

The presence of molecular motors acting as active crosslinks modifies both the mean end-to-end length $L_{0}$ of a filament strand and the stiffness $k_{e f f}$ of each elastic element. As shown earlier, an active crosslink stiffens $k_{\text {eff }}$ at high frequencies $\left(\omega>\omega_{c}\right)$, corresponding to time scales shorter than the relaxation time of the motor clusters, but longer than the relaxation time of a single filament. This correction, given in Eq. (17), is proportional to the square of the stall force $f$, which in turn is linearly proportional to the ATP activity $\Delta \mu$ (the chemical potential difference between ATP and its hydrolysis products), for small $\Delta \mu$. This will therefore yield an active stiffening of order $(\Delta \mu)^{2}$ of the shear modulus of the network. To estimate the effect of activity on $L_{0}$ we note that the network contains a bulk density $n_{\text {pass }}$ of passive crosslinks and a bulk density $n_{\text {mot }}$ of active crosslinks. Among the latter only a fraction $r=k_{o n} /\left(k_{o n}+k_{o f f}\right)$ are bound, where $k_{o n}$ and $k_{o f f}$ are the motors binding and unbinding rates, respectively. Assuming only pairwise crosslinks and no dangling ends, the mean strand length between crosslinks is $L_{0}=\frac{1}{2} \rho a /\left(r n_{\text {mot }}+n_{\text {pass }}\right)$. The rates $k_{o n}$ and $k_{o f f}$ depend on $\Delta \mu$ and are finite at chemical equilibrium when $\Delta \mu=0$. In general $r$ is expected to depend linearly on activity for small $\Delta \mu$, i.e., $r(\Delta \mu) \approx r_{0}+r_{1} \Delta \mu$. This immediately gives $L_{0} \simeq L_{0}^{(0)}+\Delta \mu L_{0}^{(1)}$, and will therefore yields active corrections to the shear modulus linear in $\Delta \mu$.

Expanding for small $\Delta \mu$, the shear modulus of the active gel will have the form $E_{\text {active }}=E_{\text {passive }}+\Delta \mu E^{(1)}+$ $(\Delta \mu)^{2} E^{(2)}+\mathcal{O}\left((\Delta \mu)^{3}\right)$. Explicit expressions for the various contributions can be obtained for instance in the limit of high frequencies using the expression (17) obtained earlier for $k_{\text {eff }}(\infty)$. The first term, $E_{\text {passive }}$, is the shear modulus of a passive cross-linked semiflexible gel $[5,6]$. The term linear in $\Delta \mu$ comes from the change in the number of crosslinks due to ATP consumption. The term quadratic in $\Delta \mu$ has contributions from the active forces (calculated in this paper), as well as smaller contributions from quadratic corrections to $r$. It can dominate at intermediate activities where it yields stiffening of the gel at high frequencies. At small $\Delta \mu$ the correction due to the variation of the binding and unbinding rates with activity will dominate. The sign of this correction is controlled by the sign of $r_{1}$ and is difficult to assess due to two competing effects. The unbinding rate $k_{\text {off }}$ is known exper- 
imentally and theoretically to increase with $\Delta \mu$ [14,21]. The binding rate $k_{\text {on }}$ is also expected to increase with $\Delta \mu$ because once a motor cluster is bound to one filament, its directed motion along the filament allows it to explore a larger region of phase space and facilitates the binding to a second filament. This can also yield stiffening of the gel at very low activity with an elastic modulus increasing linearly with $\Delta \mu$ if the increase of the binding rate with $\Delta \mu$ dominates the increase in the unbinding rate $\left(L_{0}^{(1)}<0\right.$ above). Detailed experiments are needed to address this question.

Experiments have found that the addition of active crosslinkers such as myosin II can increase the shear modulus of the network of several order of magnitudes implying that a quantitative comparison requires going into the non-perturbative regime. When the motor induced tension exceeds the bare tension, i.e., $f>\sigma_{0}$, the parts of the filaments under compression will buckle and their response will be governed solely by the bending rigidity, $\kappa$. Under such conditions, a complete calculation becomes more difficult. In the "high" frequency regime, however, the compressed parts of the filaments contribute negligibly to the force balance and the modulus is entirely controlled by the tense portion. A straightforward calculation then yields $E \simeq 2 \xi^{-2}\left(r n_{\text {mot }} / n_{\text {pass }}\right)\left(\kappa^{1 / 2} f^{3 / 2} / k_{B} T\right)+$ $\mathcal{O}\left(\frac{\sigma_{0}}{f}, \frac{\kappa}{f L_{0}^{2}}\right)$. When $r n_{\text {mot }} \sim n_{\text {pass }}$, the shear modulus of the active gel scales as $\left(f / \sigma_{0}\right)^{3 / 2}$, in agreement with observations $[14,17]$. We stress that all our results only apply if the density of bound motors is larger than a critical density required for a network of tense filaments to percolate trough the gel. For smaller values of $r n_{\text {mot }}$, one expects little effect from the active crosslinks. Conversely, if the average number motor clusters bound to each elastic unit exceeds one, our formulae also breaks down as the portion of filaments between two successive motor clusters do not experience a large tension. These arguments suggest that both motor-induced contractility and stiffening will occur only in a narrow range of density of bound motors, in qualitative agreement with experiments.

To summarise, we have studied a simplified microscopic model of a cross-linked active gel and shown that the nonlinear elasticity and collective dynamics of the motors play an important role in the macroscopic mechanical properties of the gel. In particular we show that elastic nonlinearities can lead to a gel which is contractile and stiffened by active elements above a characteristic crossover frequency due to the collective dynamics of the motors.

$$
* * *
$$

We thank Gijsje Koenderink for very useful discussions. MCM was supported by NSF grants DMR-0305407 and DMR-0705105 and by the Institut Curie in Paris through a Rotschild-Yvette-Mayent sabbatical fellowship. She thanks the Institut Curie and ESPCI for their hospitality during the completion of some of this work. TBL acknowledges the hospitality of the Institut Curie in Paris and the support of the Royal Society and the EPSRC under grant EP/E065678/1.

\section{REFERENCES}

[1] B. Alberts and A. Johnson and J. Lewis and M. RAfF and K. Roberts and P. WALter, Molecular biology of the cell (Garland, New York) 2002

[2] J. HowARD, Mechanics of motor proteins and the cytoskeleton (Sinauer, New York) 2000

[3] M.L. Gardel and J.H. Shin and F.C. MacKintosh and L. Mahadevan and P. Matsudaira and D.A. Weitz, Science, 304 (2004) 1301

[4] H. IsAmbert and A. C. Maggs, Macromolecules, 29 (1996) 1036

[5] F. Gittes and F.C. Mackintosh, Phys. Rev. E, 58 (1998) R1241

[6] D.C. Morse, Phys. Rev. E, 58 (1998) R1237

[7] K. E. Kasza and A. C. Rowata and J. Liu and T. E. AnGelini and C. P. Brangwynne and G. H. Koenderink and D. A. Weitz, Current Opinion in Cell Biology, 19 (2007) 101

[8] O. Thoumine and A. Ott, J. Cell Sci., 110 (1997) 2109

[9] B. Fabry and G. N. Maksym and J. P. Butler and M. Glogauer and D. Navajas and J. J. Fredberg, Phys. Rev. Lett., 87 (2001) 148102

[10] F. Wottawah and S. Schinkinger and B. Lincoln and R. Ananthakrishnan and M. Romeyke and J. GuCK and J. Käs, Phys. Rev. Lett., 94 (2005) 098103

[11] M. Balland and N. Desprat and D. ICARD and S. FerEOL and A. Asnacios and J. Browaeys and S. Henon and F. Gallet, Phys. Rev. E, 72 (2006) 021911

[12] N. Desprat and A. Richert and J. Simeon and A. AsNACIOS , Biophysical Journal, 88 (2005) 2224

[13] D. Stamenović, Nature Materials, 5 (2006) 597

[14] D. Mizuno and C. Tardin and C. F. Schmidt and F. C. MacKintosh, Science, 315 (2007) 370

[15] F.C. MacKintosh and A.J. Levine, Phys. Rev. Lett., 100 (2008) 18104

[16] T.B. Liverpool and A.C. Maggs, Macromolecules, 34 (2001) 6064

[17] P. M. Bendix and G. H. Koenderink and D. Cuvelier and Z. Dogic and B. Koeleman and W. M. Brieher and C. M. Field and L. Mahadevan and D. A. Weitz, Biophys. J., 107 (2008) 117960

[18] T. B. Liverpool and M. C. Marchetti, Phys. Rev. Lett., 90 (2003) 138102

[19] The condition of zero torque at the filaments' ends is strictly satisfied only on average. It suppresses filament buckling under compression.

[20] F. Jülicher and J. Prost, Phys. Rev. Lett., 75 (1995) 2618

[21] A. Parmeggiani and F. Julicher and L. Peliti and J. Prost, Europhys. Lett., 56 (4) (2001) 603 